\title{
BMJ Open Keeping Weight Off: study protocol of an RCT to investigate brain changes associated with mindfulness-based stress reduction
}

\author{
Carl Fulwiler, ${ }^{1}$ Julia A Siegel, ${ }^{2}$ Jeroan Allison, ${ }^{3}$ Milagros C Rosal, ${ }^{4}$ Judson Brewer, ${ }^{1}$ \\ Jean A King ${ }^{5}$
}

To cite: Fulwiler C, Siegel JA, Allison J, et al. Keeping Weight Off: study protocol of an RCT to investigate brain changes associated with mindfulness-based stress reduction. BMJ Open 2016;6: e012573. doi:10.1136/ bmjopen-2016-012573

- Prepublication history and additional material is available. To view please visit the journal (http://dx.doi.org/ 10.1136/bmjopen-2016012573)

Received 9 May 2016 Revised 9 September 2016 Accepted 5 October 2016

CrossMark

For numbered affiliations see end of article.

Correspondence to Dr Carl Fulwiler; carl. fulwiler@umassmed.edu

\section{ABSTRACT}

Introduction: Obesity is a growing epidemic fuelled by unhealthy behaviours and associated with significant comorbidities and financial costs. While behavioural interventions produce clinically meaningful weight loss, weight loss maintenance is challenging. This may partially be due to failure to target stress and emotional reactivity. Mindfulness-based stress reduction (MBSR) reduces stress and emotional reactivity and may be a useful tool for behaviour change maintenance. This study seeks to provide a mechanistic understanding for clinical trials of the benefits of MBSR for weight loss maintenance by examining changes in functional connectivity (FC) and the association of these changes with clinical outcomes.

Methods and analysis: Community-dwelling individuals ( $n=80$ ) who intentionally lost $\geq 5 \%$ of their body weight in the past year will be recruited and randomised to an MBSR programme or educational control. FC using resting-state functional MRI will be measured at baseline and 8 weeks. Psychological factors, health behaviours, body mass index and waist circumference will be measured at baseline, 8 weeks and 6 months post intervention. A 12-month telephone follow-up will assess self-reported weight. Analyses will characterise $\mathrm{FC}$ changes in response to MBSR in comparison with a control condition, assess the relationship between baseline FC status and pre-post MBSR changes in $\mathrm{FC}$ and investigate the association of FC change with changes in psychological factors and weight loss maintenance.

Ethics and dissemination: The University of Massachusetts Medical School Institutional Review Board has approved this study, Declaration of Helsinki protocols are being followed, and patients will give written informed consent. The Independent Monitoring Committee will monitor protocol adherence.

Results from the study will be disseminated to the medical community at conferences and submitted for publication in peer-reviewed journals when the last patient included has been followed up for 12 months.

Trial registration number: NCT02189187.
Strengths and limitations of this study

- This is a timely and innovative study that will advance the field of mindfulness research and increase understanding of its mechanisms of action, setting the stage for a comprehensive clinical trial.

- The intervention is low-risk, highly accessible, low-cost and is implemented in a real-world clinical population and community setting. The control course was specifically designed to serve as an attention control for mindfulness-based stress reduction.

- The cost of a neuroimaging biomarker could be considered a limitation for large-scale clinical trials, but the insights about neural mechanisms of change cannot be obtained with other methods.

\section{INTRODUCTION}

Unhealthy behaviours such as overeating and sedentary lifestyles are major contributors to cardiovascular disease, cancer, type 2 diabetes and other chronic conditions. They have produced a rapid rise in obesity that threatens to reverse recent gains in life expectancy ${ }^{1}$ and accounts for a large percentage of premature deaths in the USA. ${ }^{2}$ The number of obese adults in the USA is expected to rise by 65 million from 2010 to 2030 , resulting in a predicted additional $6-8$ million cases of diabetes, 5-6.8 million cases of heart disease and stroke, and over 400000 cancer cases. ${ }^{3}$ For each $5 \mathrm{~kg} / \mathrm{m}^{2}$ increase in Body Mass Index (BMI), the risks of oesophageal cancer and colon cancer in men increase by $52 \%$ and $24 \%$, respectively, and the risks of endometrial cancer, gall bladder cancer, and breast cancer in women increase by $59 \%, 59 \%$ and $12 \%$, respectively. ${ }^{4}$

In addition to significant morbidity, obesity has a substantial financial impact due to 
healthcare costs and decreased productivity. Obesity-related US healthcare costs were $\$ 2.5$ trillion in 2009 and are predicted to rise by at least $\$ 22$ billion/ year by 2020 and $\$ 48$ billion/year by $2030 .^{3}$

Weight loss is vital for reducing these extensive health and economic burdens, and even minimal weight loss has a meaningful impact. In an overweight and obese population in Ireland, a $1 \mathrm{~kg} / \mathrm{m}^{2}$ decrease in BMI led to 26 fewer cases of chronic disease per 1000 men and 28 fewer cases per 1000 women. ${ }^{5}$ Similarly, a $1 \%$ decrease in BMI across the US population ( $1 \mathrm{~kg}$ weight loss for the average adult) is predicted to prevent 2.1-2.4 million cases of diabetes, 1.4-1.7 million cases of cardiovascular disease, and 73 000-127000 cancer cases; and would only require reducing caloric intake by $20 \mathrm{kcal} /$ day for 3 years. ${ }^{3}$

Current obesity treatments include lifestyle modification, pharmacotherapy and surgical options. A systematic review of various approaches to weight loss maintenance found that behavioural interventions involving food intake and physical activity led to significant, albeit small, improvements in weight loss maintenance at 12 months after the intervention. ${ }^{6}$ Exercise programmes alone, however, may be most effective in the adoption phase. ${ }^{7}$ Similarly, certain medications such as orlistat and sibutramine facilitate weight loss but commonly only work short term when used alone, and may have unfavourable side effects. ${ }^{8}$ Bariatric surgery can be effective long term, but it can be associated with perioperative surgical risks and weight regain is common. ${ }^{8}$ Also, many patients are unwilling or ineligible to undergo surgery.

Many interventions are effective for initiating weight loss and other health behaviour changes, but they have shown only limited ability to affect significant, long-term behaviour change in the majority of adults. ${ }^{7-11}$ In part, this may be attributable to a failure of existing interventions to adequately address the effects of stress and emotional reactivity on relapse to unhealthy behaviours and failure to maintain long-term behaviour change. Perceived stress and symptoms of emotional reactivity (depression, anxiety, anger) are linked to unhealthy lifestyle behaviours ${ }^{12-15}$ and predict worse outcomes in maintenance studies. ${ }^{16-18}$ Indeed, studies of health behaviour change have demonstrated that perceived stress ${ }^{19-21}$ and indices of emotional reactivity such as anxiety, ${ }^{22-24}$ depression and anger ${ }^{25-27}$ are associated with poor outcomes. In contrast, positive affect is associated with improved outcomes. ${ }^{28} 29$

Mindfulness, defined as paying attention to one's inner and outer experiences in a non-judgmental manner from moment to moment, ${ }^{30}$ has been associated with healthy behaviours. Dispositional mindfulness in obese patients awaiting bariatric surgery was found to be positively associated with a restrained eating style (using restrictive control over food to lose weight) but negatively associated with emotional (eating in response to emotional states) and external eating behaviours (eating in response to external cues). Mindfulness may discourage external eating by increasing sensitivity to hunger and satiety such that these internal cues guide behaviour instead. In addition, mindfulness may prevent emotional eating by encouraging acceptance of negative feelings, lowering stress and thus promoting distinction between emotion and hunger. Finally, mindfulness has been shown to decrease impulsivity which may reduce unhealthy eating behaviours. $^{31}$

Several mindfulness-based or mindfulness-associated practices are promising agents of behaviour change, but we need to understand their neural mechanisms in order to optimise their use. Mindfulness-based stress reduction (MBSR) is a psycho-educational programme that teaches emotional and physical self-care. Participants receive training in formal and informal mindfulness practices and learn about the role of good nutrition, rest and exercise, as well as the role played by thoughts and emotions in physical and emotional health. They are taught how to cultivate a non-reactive awareness of mental and physical experience in an effort to increase self-efficacy and reduced emotional reactivity-leading to healthier lifestyle practices. A recent comparative effectiveness review found moderately strong evidence for mindfulness meditation programmes, particularly MBSR, for anxiety, depression and pain compared with non-specific active controls, and weaker evidence for stress and health-related quality of life. ${ }^{32}$ Evidence is mixed regarding the effectiveness of mindfulness-based interventions on weight loss, at least with relatively short follow-up periods. ${ }^{33}$ Whether MBSR can support maintenance of weight loss following successful initiation of health behaviour change warrants investigation based on its ability to lower emotional and behavioural reactivity to stress and negative emotions, risk factors for relapse to unhealthy behaviours. Importantly, an understanding of neural targets and mechanisms of change is necessary for specifying for whom mindfulness is likely to work and for optimising the intervention for maximal effectiveness. Mindfulness may work better in specific subpopulations, as seen in a group of women with specific endogenous opioidergic activity who were found to be more receptive to mindfulness training in an effort to decrease pleasure eating. ${ }^{34}$ Additionally, efficacy of mindfulness-based therapy has been strongly positively associated with dispositional mindfulness of participants and therapists. ${ }^{35}$ Neuroimaging, specifically resting-state functional MRI (fMRI), is a powerful approach to identifying mechanisms of change for MBSR involving the role of emotion regulation in maintenance of health behaviour change.

Neuroimaging studies report an association between MBSR and changes in functional connectivity (FC) that may reflect improved attention, sensory processing and reflective awareness of sensory experience. ${ }^{36}{ }^{37}$ Mindfulness has also been shown to alter resting-state FC of the amygdala, a region involved in physiological 
stress response. A randomised controlled trial (RCT) found that a 3-day intensive mindfulness training reversed the effects of stress on the amygdala-subgenual anterior cingulate cortex in a group of stressed unemployed adults in the community. ${ }^{38}$ This mindfulness training was also shown to increase resting-state FC between the default mode network and the left dorsolateral prefrontal cortex, an area involved in top-down executive control. ${ }^{39}$ However, no previous studies have examined how mindfulness training affects the neural circuitry of emotion regulation in a weight loss sample and little is known about mechanisms of behaviour change in people undergoing mindfulness training.

\section{Gaps in knowledge}

Emerging evidence suggests that mindfulness may be helpful for changing behaviours such as overeating ${ }^{40}$ but the mechanistic knowledge of how mindfulness facilitates behaviour change is not known. Efforts to fill this gap could enhance our ability to identify likely 'responders' and thus optimise intervention efforts, consistent with current trends towards personalised medicine approaches. Specifically, we are lacking knowledge of specific neural targets of mindfulness training to inform clinical trials of health behaviour change and maintenance of change. In addition, data on long-term outcomes of mindfulness training are lacking.

Understanding the neural mechanisms that link MBSR to changes in emotional regulation and behaviour are a critical next step in tapping the potential of MBSR as an intervention for behaviour change and maintenance. A validated biomarker will allow investigators to monitor fidelity of intervention delivery, adherence and doseresponse in clinical trials. If validated as a biomarker, changes in FC will allow future studies to determine characteristics of individuals who are most responsive to MBSR and which components of the mindfulness intervention are most active, and may enable development of a more compact and potent intervention. FC may also help identify subsets of high-risk patients that would benefit from specific tailoring of the intervention. It is worth noting that if clinical trials prove that MBSR prevents weight regain, MRIs would not be required in a larger dissemination study or as the intervention is deployed in a large-scale public health approach.

\section{Study aims and hypotheses}

To characterise FC, psychological, behavioural and anthropometric changes in response to MBSR and the comparison condition, we will randomise a sample of 80 participants who have intentionally lost $\geq 5 \%$ of their body weight during the previous year to MBSR or an attention control specifically designed to be structurally equivalent to MBSR. Study aims and hypotheses are as follows.

Our first primary aim is to characterise FC changes in response to MBSR and the comparison condition. We hypothesise that participants randomised to the MBSR condition will experience greater increases in FC from baseline to post intervention (Hypothesis 1), and participants with higher baseline FC will show less change in response to MBSR (Hypothesis 2).

Our second primary aim is to investigate the association of FC change with changes in psychological factors and maintenance of weight loss at 8 weeks and 6-month follow-up. We hypothesise that increases in FC will be associated with improvement in depressive symptoms (Hypothesis 3) and inversely related to decreased weight (BMI) and total waist circumference (Hypothesis 4).

Our third primary aim is to assess changes in BMI at 6 and 12 months to obtain preliminary measures of effect size and variability by study group for future clinical trials.

As secondary aims, we will use mediation analysis to determine how change in FC is explained by (1) class attendance, (2) self-reported time in homework practice, (3) self-reported time for each specific component of the multifaceted training programme and (4) trait mindfulness. An exploratory aim is to examine correlations of change in FC with changes in additional psychological factors (perceived stress, trait anger, trait anxiety, positive affect) and health behaviours (healthy eating, physical activity, sleep quality).

\section{METHODS}

\section{Study design}

The 'Keeping Weight Off' study is a randomised, prospective, two-armed, controlled trial. A sample of 80 participants from the community, who have intentionally lost at least $5 \%$ of their body weight during the previous year, will be equally randomised into two groups: an MBSR programme and a healthy living course (HLC) an attention control specifically designed to be structurally equivalent to MBSR. ${ }^{41}$ The HLC uses the same format of 8-weekly classes lasting 2.5 hours, and controls for attention and other non-specific factors including staff interactions, psychoeducation about health and stress management, classroom format, homework, group process and data collection. ${ }^{41}$ Our main outcome measures are resting-state FC, depression symptoms, BMI and waist circumference. FC, psychological factors, health behaviours, BMI and waist circumference will be measured at baseline and 8 weeks. Psychological factors, health behaviours, BMI and waist circumference will also be measured at 6 months. In addition, a telephone follow-up will be attempted on participants at 12 months to assess weight.

Total planned enrolment is 80 participants. Screening for eligibility criteria, baseline visits and follow-up visits will take place at University of Massachusetts Medical School (UMMS), Worcester, Massachusetts. All MBSR classes will be conducted at the UMMS Center for Mindfulness in Shrewsbury, Massachusetts.

\section{Study participants}

We will recruit participants who range in age from 25 to 60 (chosen to minimise age-related changes in FC), 
Table 1 Eligibility criteria

Inclusion criteria:
Men and women

3D, three dimensional; BMI, Body Mass Index; FFE, Turbo Flash Field Echo; MBSR, mindfulness-based stress reduction; T1W, T1 weighted; TFE, Turbo Field Echo.

have intentionally lost $\geq 5 \%$ of their body weight during the previous year and are motivated to maintain this weight loss. Individuals will be excluded if they have participated in an MBSR course, regular meditation practice or any other form of meditative practice (such as yoga, Tai Chi or contemplative prayer), for more than an average of 20 min per week within the past 2 years. Full inclusion and exclusion criteria are listed in table 1.

Eligible participants will proceed with the baseline visit prior to randomisation, including collection of demographic information about age, education, marital status, occupation and current employment status, followed by questionnaires and fMRI scan. The full study schedule of recruitment, treatment and assessments is described in table 2. All participants will provide informed consent. This process will be conducted by the study coordinator in person prior to the baseline visit, following the protocol established by the University of Massachusetts Institutional Review Board (IRB). Study staff will keep in touch with participants regarding attendance and homework completion and provide support and encouragement to continue with the intervention if participants express dissatisfaction. Participants who drop out of the intervention will be asked to return for all follow-up visits to complete all outcome data collection.

\section{Study recruitment}

Recruitment will be conducted using advertising in the community (internet, flyers and social media) of Worcester County, which has a population of over 800 000, as well as a two-stage process to recruit outpatients from the UMass Memorial Medical Center using the electronic medical record. The largest healthcare system in Central and Western Massachusetts, the medical centre has a large population from which to draw, with nearly 70000 patient visits for primary care alone, and a Weight Center that sees nearly 1000 new patients per year. Our team has an excellent record of recruitment and retention in our previous overweight/obese subjects using these methods.

First, with a Health Insurance Portability and Accountability Act (HIPAA) waiver authorisation a search query will be conducted with basic eligibility criteria, and second, identified records will be reviewed to exclude causes of weight loss such as serious illness or weight loss medications. Web-based and telephone screening will determine whether they meet inclusion criteria. Further screening will be done in person to gather a medical history and complete the Structured Clinical Interview for DSM-IV (SCID) to exclude participants with a serious psychiatric, cognitive or medical disorder or a history of alcohol or substance abuse or dependence in the past 6 months.

\section{Randomisation and study blinding}

Study participants will be equally randomised to either the MBSR intervention arm or HLC arm based on a permuted blocks randomisation scheme. In this procedure, treatment allocations will be made within blocks so that the numbers assigned to each arm are equal after each block has been filled. Blocks of various sizes $(2,4,6)$ will be used 
Table 2 Study schedule of recruitment, treatment and assessments as a function of time points (according to the SPIRIT 2013 figure guidelines)

\begin{tabular}{|c|c|c|c|c|c|}
\hline \multirow[b]{2}{*}{ TIME POINT } & \multicolumn{5}{|c|}{ Duration of the study } \\
\hline & $\begin{array}{l}\text { Recruitment } \\
t_{0}\end{array}$ & $\begin{array}{l}\text { Baseline } \\
t_{1}\end{array}$ & $\begin{array}{l}\text { Post-treatment } \\
\text { (8 weeks) } \\
t_{2}\end{array}$ & $\begin{array}{l}\text { Follow-up } \\
\text { (6 months) } \\
t_{3}\end{array}$ & $\begin{array}{l}\text { Follow-up } \\
\text { (12 months) } \\
t_{4}\end{array}$ \\
\hline \multicolumn{6}{|l|}{ RECRUITMENT } \\
\hline $\begin{array}{l}\text { Screening for inclusion/exclusion } \\
\text { criteria }\end{array}$ & $x$ & & & & \\
\hline Informed consent & & $\mathrm{x}$ & & & \\
\hline Assignment to treatment arms & $\mathrm{x}$ & & & & \\
\hline \multicolumn{6}{|l|}{ TREATMENT } \\
\hline MBSR & & $\mathrm{x}$ & $\mathrm{x}$ & & \\
\hline HLC & & $\mathrm{x}$ & $\mathrm{x}$ & & \\
\hline \multicolumn{6}{|l|}{ ASSESSMENT } \\
\hline SCID-IV & $\mathrm{x}$ & & & & \\
\hline fMRI & & $\mathrm{x}$ & $\mathrm{x}$ & & \\
\hline Weight & & $\mathrm{x}$ & $\mathrm{x}$ & $\mathrm{x}$ & $\mathrm{x}$ \\
\hline Height (and BMI) & & $\mathrm{x}$ & $\mathrm{x}$ & $\mathrm{x}$ & \\
\hline Waist circumference & & $\mathrm{x}$ & $\mathrm{x}$ & $\mathrm{x}$ & \\
\hline CES-D & & $\mathrm{x}$ & $\mathrm{x}$ & $\mathrm{x}$ & \\
\hline PSS-14 & & $\mathrm{x}$ & $\mathrm{x}$ & $\mathrm{x}$ & \\
\hline STAI-T & & $\mathrm{x}$ & $\mathrm{x}$ & $\mathrm{x}$ & \\
\hline STAXI-II & & $\mathrm{x}$ & $\mathrm{x}$ & $\mathrm{x}$ & \\
\hline Satisfaction with life & & $\mathrm{x}$ & $\mathrm{x}$ & $\mathrm{x}$ & \\
\hline Emotion Regulation & & $x$ & $x$ & $x$ & \\
\hline \multicolumn{6}{|l|}{ Questionnaire } \\
\hline Eating Behaviour Inventory & & $\mathrm{x}$ & $x$ & $\mathrm{x}$ & \\
\hline $\begin{array}{l}\text { Internal Disinhibition Subscale of } \\
\text { the Eating Inventory }\end{array}$ & & $x$ & $\mathrm{x}$ & $x$ & \\
\hline Paffenbarger Physical Activity & & $\mathrm{x}$ & $\mathrm{x}$ & $\mathrm{x}$ & \\
\hline \multicolumn{6}{|l|}{ Scale } \\
\hline Pittsburgh Sleep Quality Index & & $\mathrm{x}$ & $\mathrm{x}$ & $\mathrm{x}$ & \\
\hline
\end{tabular}

in random order, to facilitate allocation concealment, that is, to make it nearly impossible to determine the treatment assignment based on a pattern of previous treatment allocations. Randomisation will be implemented using sealed envelopes by the study coordinator who will be the only member of the research team who is not blind to treatment assignment. A unique identification number will help to ensure that blindness is maintained throughout the study. All members of the research team involved in data analysis will be blind to treatment assignment.

To address the possibility that an imbalanced distribution of baseline FC could mask important findings, we will employ a non-stratified permuted-block randomised design with an interim analysis of the distribution of baseline FC. If important imbalances are found, we will implement recruitment strategies to increase enrolment of participants who have baseline FC with a specified range combined with covariate-adaptive randomisation techniques. Residual imbalances will be addressed with post hoc statistical adjustment.

\section{Assessments}

Resting-state FC

In contrast to task-evoked functional and effective connectivity studies, resting-state fMRI enables examination of the brain's intrinsic functional connections in the absence of externally controlled stimuli or tasks. ${ }^{42}{ }^{43} \mathrm{FC}$ is responsive to changing levels of stress, ${ }^{44}$ intense training on a task ${ }^{45}$ and recently, meditation practice including MBSR. ${ }^{46}{ }^{47} \mathrm{FC}$ has been shown to have remarkable consistency and moderate-to-high test-test reliability over periods of months to a year as well. ${ }^{48} 49$ All MRIs will be acquired on the 3T scanner (Philips Achieva) in the UMMS Advanced MRI Center. Three-dimensional highresolution structural T1-weighted MR images will be obtained to provide anatomical landmarks. Following the structural imaging, resting-state fMRI data will be collected. Participants will be instructed to remain relaxed with eyes closed as fMRI images are continuously collected for $10 \mathrm{~min}$. The duration of the entire MRI procedure will be $30 \mathrm{~min}$. 
Psychological symptoms

The Center for Epidemiologic Studies Depression Scale $(\text { CES-D })^{50}$ depression symptom score is a primary outcome. Additional outcomes include perceived stress, anxiety, anger, positive affect and emotion regulation, which will be assessed using the following measures, respectively: the Perceived Stress Scale (PSS-14), ${ }^{51}$ the State-Trait Anxiety Inventory-trait version (STAI-T), ${ }^{52}$ the State-Trait Anger Expression Inventory-II (STAXI-II) trait anger subscale ${ }^{53}$ the Satisfaction with Life Scale ${ }^{54}$ and the Emotion Regulation Questionnaire. ${ }^{55}$

\section{Health behaviours}

Health behaviours will be assessed using the Eating Behaviour Inventory, ${ }^{56}$ the Internal Disinhibition Subscale of the Eating Inventory, ${ }^{57}$ the Paffenbarger Physical Activity Scale ${ }^{58}$ and the Pittsburgh Sleep Quality Index (PSQI), ${ }^{59}$ respectively.

\section{Anthropometrics}

Procedures are adapted from the Centers for Disease Control (CDC)'s Anthropometry Procedures Manual. ${ }^{60}$ For the measurement of height and weight, the Seca 213 Portable stadiometer and the tanita, BWB- 800 electronic scale will be used and measurements will be taken twice and averaged to assure reliability. Measurement of waist circumference will be taken directly on the skin using measuring tape placed just above top of the iliac crest on each side.

\section{Potential mediators and confounders}

Intervention engagement will be assessed with class attendance and a home practice log. Treatment expectancy and credibility will be assessed with the Credibility/ Expectancy Questionnaire (CEQ $)^{61}$ modified slightly to substitute the word 'Class' for 'Therapy' in the instructions. Secondary analyses will examine how change in FC is explained by (1) class attendance, (2) self-reported time in homework practice, (3) self-reported time for each specific component of the multifaceted training programme and (4) trait mindfulness as measured by the Five Facet Mindfulness Questionnaire (FFMQ) ${ }^{62}$

\section{Interventions}

Experimental condition: MBSR

MBSR, described above, is taught in eight weekly classes and one all-day retreat. Homework assignments include formal meditation practices and informal practices during daily life. Classroom activities teach formal meditation practices including sitting meditation, body scan, mindful yoga and walking meditation. Participants also learn to bring awareness in the present moment to aspects of daily life and apply this practice to stressful experiences in order to avoid reflexive or conditioned reactions. The goal of MBSR is to provide participants with these skills for lifelong self-management.

The MBSR classes are taught by certified teachers from the UMass Center for Mindfulness. All teachers have completed the rigorous training and certification for MBSR through the Center for Mindfulness. To further assure fidelity, each class will be reviewed in weekly sessions with a coinvestigator. In addition, the principal investigators will routinely convene the entire clinical research team to monitor the overall fidelity of the MBSR intervention.

\section{Attention control: HLC}

The HLC was specifically designed to serve as a control condition for MBSR. ${ }^{41}$ HLC consists of eight weekly 2-hour classes. Sessions consist of lectures and discussion on the following topics: healthy living, healthy eating, physical activity and health, sleep and health, stress management, time management and unhealthy behaviours (smoking, drinking). The HLC is designed to control for attention and other non-specific factors including staff interactions, psychoeducation about health and stress management, classroom format, homework, group process and data collection. ${ }^{41}$

\section{Data collection}

Electronic data capture (EDC, Research Electronic Data Capture (REDCap)) will be used for all data collection except imaging. Surveys for psychological symptoms, health behaviours and potential mediators and confounders are programmed into the EDC system. During research visits, participants will enter survey responses directly into this system and research staff will enter screening and anthropometric data with periodic checks for quality assurance. All data will be maintained in a secure location accessible only to study personnel. For MRI measures, data will be analysed blind to intervention group by two fMRI experts who perform cross-validity checks.

\section{Data analysis and sample size considerations}

We based the study sample size on Hypothesis 1, powered to detect an effect size of 0.32 SDs. This moderate Cohen effect size ${ }^{63}$ represents differential overtime change for the intervention versus comparison group cast in SD units. The study design calls for a baseline measurement and two follow-up measurements. In addition, we assumed a two-tailed $\alpha$ error of 0.05 , and a within-person correlation for overtime measurement of $0.8 .^{64}$ With these assumptions, 40 participants in each group are needed for $80 \%$ power. We will enrol 100 participants (50 randomised to each arm) and allow for up to $20 \%$ loss to follow-up, for an effective sample size of 80 .

Statistical analysis will begin with univariate summaries of data distributions and examination of longitudinal trends with graphic displays. Bivariate associations will then be examined using the $\chi^{2}$ test, analysis of variance (ANOVA), and the Spearman correlation test. Hypotheses 1 and 2 focus on overtime differences between the two study groups, while Hypotheses 2 and 3 focus on overtime differences for the combined sample. Hypotheses will be formally tested using generalised linear mixed models that represent the clustering of 
observations within participants as a random intercept, adopt an appropriate link and distribution for the specific outcome and parameterise the intervention effect as a group-time intervention, when appropriate. ${ }^{65}$ To preserve the power of randomisation, hypothesis testing will be performed on an intent-to-treat basis. As a secondary aim, mediation analysis will determine how change in FC is explained by markers of intervention adherence using techniques as described by MacKinnon. ${ }^{66}{ }^{67}$ Sensitivity analyses with multiple imputation with chained equations will address missing data.

Imaging data is preprocessed using Statistical Parametric Mapping (SPM8) software (Wellcome Department of Cognitive Neurology, London, UK) running under the MATLAB environment (Mathworks, Sherborn, Massachusetts, USA). The data are initially corrected for motion (threshold of $2 \mathrm{~mm}$ ). Further preprocessing of the data includes (1) slice scan time correction, (2) spatial smoothing using a three-dimensional Gaussian filter (4-mm full width at half maximum (FWHM)) and (3) voxel-wise linear detrending and $0.01-0.08 \mathrm{~Hz}$ band-pass filtering. Structural and functional data of each participant are then be transformed to standard stereotaxic space ${ }^{68}$ to facilitate group analysis.

Following the preprocessing steps, FC will be generated using correlational analysis. Left and right amygdalae are used as separate seed regions of interest (ROI). FC maps from each individual seed will then be calculated for each individual subject using Resting-State fMRI Data Analysis Toolkit (REST, http://www.restfmri. net). Each seed ROI will be evaluated using two-way repeated measures ANOVA on a voxel-by-voxel basis (factors: group and imaging day) at the threshold of $\mathrm{p}<0.05$ after accounting for multiple comparisons using the criteria of false discovery rate (FDR). ${ }^{69}$ Voxels with significantly changed FC within each ROI will then be averaged to generate the FC change for the ROI. This procedure will yield a single value of amygdala-orbitofrontal FC for each participant at each point in time. All ROI definitions are based on Automated Anatomical Labelling (AAL) ${ }^{70}$ built in the MARSeille Boîte À Région (MarsBAR) toolbox of SPM8. Image analysis will be done blind to group membership.

As a secondary analysis, we will explore FC changes in other brain networks following MBSR using other seed regions. For example, other cortical regions that have been implicated in modulation of limbic reactivity (eg, anterior cingulate) will be examined using a hypothesisdriven approach.

\section{Ethical considerations and dissemination plans}

This study is registered as a national clinical trial (NCT02189187) and Declaration of Helsinki protocols are being followed, and patients will give written informed consent. Protocol adherence will be monitored by the Independent Monitoring Committee (for full WHO Trial Registration Data Set information see online supplementary appendix). Results from the study will be disseminated to the medical community at conferences and submitted for publication in peer-reviewed journals when the last patient included has been followed up for 12 months. Negative and inconclusive as well as positive results will be published or made publicly available through the study website http://www. umassmed.edu/keepingweightoff.

Author affiliations

${ }^{1}$ Departments of Psychiatry and Medicine and Center for Mindfulness, University of Massachusetts Medical School, Shrewsbury, Massachusetts, USA

${ }^{2}$ University of Massachusetts Medical School, Worcester, Massachusetts, USA ${ }^{3}$ Department of Quantitative Health Sciences, University of Massachusetts Medical School, Worcester, Massachusetts, USA

${ }^{4}$ Department of Medicine, University of Massachusetts Medical School, Worcester, Massachusetts, USA

${ }^{5}$ Departments of Psychiatry, Neurology and Radiology, University of Massachusetts Medical School, Worcester, Massachusetts, USA

Acknowledgements The authors thank Dr Nanyin Zhang and Dr Wei Huang for technical assistance with study design. We also extend our sincere gratitude to Dr Saki Santorelli, Executive Director of the Center for Mindfulness at the University of Massachusetts Medical School, for his support and contributions, and the staff and teachers of the Center for Mindfulness for their assistance. The project was conducted at the UMMS Advanced MR Imaging Center with support from Dr Shaokuan Zheng.

Contributors CF conceptualised the study, and JAK, JA, MCR and JB participated in the study design. CF, JAK, and JAS collected data. CF and JAS wrote and all authors revised the article and approved the final version to be published.

Funding The National Center for Complementary and Integrative Health (NCCIH)) provided funding for this trial (grants R34AT006963 and R34 AT006963-01A1 to CF and JAK). The design of this trial was reviewed and approved by NCCIH's Office of Clinical and Regulatory Affairs. Support was also provided by an award from the UMMS Department of Radiology.

Disclaimer The content is solely the responsibility of the authors and does not necessarily represent the official views of the National Institutes of Health or the UMMS Department of Radiology Advanced MR Imaging Center.

Competing interests None declared.

Ethics approval University of Massachusetts Medical School Institutional Review Board.

Provenance and peer review Not commissioned; externally peer reviewed.

Data sharing statement For investigators expressing interest in data analyses, we will welcome proposals for consideration by the study investigators. If there is no overlap in research interests, necessary data files will be generated and provided, and writing groups identified. We are sensitive to the potential for identification of participants and loss of confidentiality. Consequently, certain sensitive types of data may not be released. However, in these cases analyses may be performed by our group and sent to the investigators for interpretation.

Open Access This is an Open Access article distributed in accordance with the Creative Commons Attribution Non Commercial (CC BY-NC 4.0) license, which permits others to distribute, remix, adapt, build upon this work noncommercially, and license their derivative works on different terms, provided the original work is properly cited and the use is non-commercial. See: http:// creativecommons.org/licenses/by-nc/4.0/

\section{REFERENCES}

1. Ogden C, Carroll M. Prevalence of overweight, obesity, and extreme obesity among adults: United States, trends 1976-1980 through 2007-2008. CDC Natl Cent Heal Stat. 2010. http://www.cdc.gov/ nchs/data/hestat/obesity_adult_11_12/obesity_adult_11_12.htm 
2. Schroeder SA. We can do better--improving the health of the American people. N Engl J Med. 2007;357:1221-8.

3. Wang YC, McPherson K, Marsh T, et al. Health and economic burden of the projected obesity trends in the USA and the UK. Lancet. 2011;378:815-25.

4. Renehan AG, Tyson M, Egger M, et al. Body-mass index and incidence of cancer: a systematic review and meta-analysis of prospective observational studies. Lancet. 2008;371:569-78.

5. Kearns K, Dee A, Fitzgerald AP, et al. Chronic disease burden associated with overweight and obesity in Ireland: the effects of a small BMI reduction at population level. BMC Public Health. 2014;14:143.

6. Dombrowski SU, Knittle K, Avenell A, et al. Long term maintenance of weight loss with non-surgical interventions in obese adults: systematic review and meta-analyses of randomised controlled trials. BMJ. 2014;348:92646.

7. Marcus BH, Williams DM, Dubbert PM, et al. What we know and what we need to know: a scientific statement from the American Heart Association Council on Nutrition, Physical Activity, and Metabolism (Subcommittee on Physical Activity); Council on Cardiovascular Disease in the Young; and the Interdisciplinary Working Group on Quality of Care and Outcomes Research. Circulation. 2006;114:2739-52.

8. Hainer V, Toplak H, Mitrakou A. Treatment modalities of obesity: what fits whom? Diabetes Care. 2008;31(Suppl 2):S269-77.

9. Hajek P, Stead LF, West R, et al. Relapse prevention interventions for smoking cessation. Cochrane Database Syst Rev. 2009;(1): CD003999.

10. Merrill RM, Aldana SG, Greenlaw RL, et al. Can newly acquired healthy behaviors persist? An analysis of health behavior decay Prev Chronic Dis. 2008;5:A13.

11. Ory MG, Lee Smith M, Mier N, et al. The science of sustaining health behavior change: the health maintenance consortium. Am $J$ Health Behav. 2010;34:647-59.

12. Björntorp P, Rosmond R. Obesity and cortisol. Nutrition. 2000;16:924-36.

13. Lattimore P, Maxwell L. Cognitive load, stress, and disinhibited eating. Eat Behav. 2004;5:315-24.

14. O'Connor DB, Jones F, Conner M, et al. Effects of daily hassles and eating style on eating behavior. Health Psychol. 2008;27:S20-31.

15. Wallis DJ, Hetherington MM. Emotions and eating. Self-reported and experimentally induced changes in food intake under stress. Appetite. 2009:52:355-62.

16. Cosci F, Pistelli F, Lazzarini N, et al. Nicotine dependence and psychological distress: outcomes and clinical implications in smoking cessation. Psychol Res Behav Manag. 2011:4:119-28.

17. Elfhag $\mathrm{K}$, Rössner $\mathrm{S}$. Who succeeds in maintaining weight loss? A conceptual review of factors associated with weight loss maintenance and weight regain. Obes Rev. 2005;6:67-85.

18. Wing RR, Papandonatos G, Fava JL, et al. Maintaining large weight losses: the role of behavioral and psychological factors. J Consult Clin Psychol. 2008;76:1015-21.

19. Evers KE, Prochaska JO, Johnson JL, et al. A randomized clinical trial of a population- and transtheoretical model-based stress-management intervention. Heal Psychol. 2006;25:521-9.

20. Mansyur CL, Pavlik VN, Hyman DJ, et al. Self-efficacy and barriers to multiple behavior change in low-income African Americans with hypertension. J Behav Med. 2013;36:75-85.

21. $\mathrm{Ng}$ DM, Jeffery RW. Relationships between perceived stress and health behaviors in a sample of working adults. Health Psychol. 2003;22:638-42.

22. Benninghoven D, Kaduk A, Wiegand U, et al. Influence of anxiety on the course of heart disease after acute myocardial infarction-Risk factor or protective function? Psychother Psychosom. 2006;75:56-61.

23. Gariepy G, Nitka D, Schmitz N. The association between obesity and anxiety disorders in the population: a systematic review and meta-analysis. Int J Obes. 2010;34:407-19.

24. Kuhl EA, Fauerbach JA, Bush DE, et al. Relation of anxiety and adherence to risk-reducing recommendations following myocardial infarction. Am J Cardiol. 2009;103:1629-34.

25. Castro Y, Kendzor DE, Businelle MS, et al. Structural and predictive equivalency of the Wisconsin smoking withdrawal scale across three racial/ethnic groups. Nicotine Tob Res. 2011;13:548-55.

26. Kahler CW, Strong DR, Niaura R, et al. Hostility in smokers with past major depressive disorder: relation to smoking patterns, reasons for quitting, and cessation outcomes. Nicotine Tob Res. 2004;6:809-18.

27. Patterson F, Kerrin K, Wileyto EP, et al. Increase in anger symptoms after smoking cessation predicts relapse. Drug Alcohol Depend. 2008;95:173-6.

28. Ogedegbe GO, Boutin-Foster C, Wells MT, et al. A randomized controlled trial of positive-affect intervention and medication adherence in hypertensive African Americans. Arch Intern Med. 2012;172:322-6.

29. Peterson JC, Charlson ME, Hoffman Z, et al. A randomized controlled trial of positive-affect induction to promote physical activity after percutaneous coronary intervention. Arch Intern Med. 2012;172:329-36.

30. Kabat-Zinn J. Mindfulness-based interventions in context: past, present, and future. Clin Psychol Sci Pract. 2003;10:144-56.

31. Ouwens MA, Schiffer AA, Visser LI, et al. Mindfulness and eating behaviour styles in morbidly obese males and females. Appetite. 2015;87:62-7.

32. Goyal M, Singh S, Sibinga EMS, et al. Meditation programs for psychological stress and well-being: a systematic review and meta-analysis. JAMA Intern Med. 2014;174:357-68.

33. Fulwiler C, Brewer JA, Sinnott S, et al. Mindfulness-based interventions for weight loss and CVD risk management. Curr Cardiovasc Risk Rep. 2015;9.

34. Mason AE, Lustig RH, Brown RR, et al. Acute responses to opioidergic blockade as a biomarker of hedonic eating among obese women enrolled in a mindfulness-based weight loss intervention trial. Appetite. 2015;91:311-20.

35. Khoury B, Lecomte T, Fortin G, et al. Mindfulness-based therapy: a comprehensive meta-analysis. Clin Psychol Rev. 2013;33: 763-71.

36. Roland LT, Lenze EJ, Hardin FM, et al. Effects of mindfulness based stress reduction therapy on subjective bother and neural connectivity in chronic tinnitus. Otolaryngol Head Neck Surg. 2015;152:919-26.

37. Kilpatrick LA, Suyenobu BY, Smith SR, et al. Impact of mindfulness-based stress reduction training on intrinsic brain connectivity. Neuroimage. 2011;56:290-8.

38. Taren AA, Gianaros PJ, Greco CM, et al. Mindfulness meditation training alters stress-related amygdala resting state functional connectivity: a randomized controlled trial. Soc Cogn Affect Neurosci. 2015;10:1758-68.

39. Creswell JD, Taren AA, Lindsay EK, et al. Alterations in resting state functional connectivity link mindfulness meditation with reduced interleukin-6: a randomized controlled trial. Biol Psychiatry. 2016;80:53-61.

40. Katterman SN, Kleinman BM, Hood MM, et al. Mindfulness meditation as an intervention for binge eating, emotional eating, and weight loss: a systematic review. Eat Behav. 2014;15:197-204.

41. Pbert L, Madison JM, Druker S, et al. Effect of mindfulness training on asthma quality of life and lung function: a randomised controlled trial. Thorax. 2012;67:769-76.

42. Biswal B, Yetkin FZ, Haughton VM, et al. Functional connectivity in the motor cortex of resting human brain using echo-planar MRI. Magn Reson Med. 1995;34:537-41.

43. Fox MD, Raichle ME. Spontaneous fluctuations in brain activity observed with functional magnetic resonance imaging. Nat Rev Neurosci. 2007;8:700-11.

44. Liston C, McEwen BS, Casey BJ. Psychosocial stress reversibly disrupts prefrontal processing and attentional control. Proc Natl Acad Sci USA 2009;106:912-17.

45. Lewis CM, Baldassarre A, Committeri $\mathrm{G}$, et al. Learning sculpts the spontaneous activity of the resting human brain. Proc Natl Acad Sci USA 2009;106:17558-63.

46. Brewer JA, Worhunsky PD, Gray JR, et al. Meditation experience is associated with differences in default mode network activity and connectivity. Proc Natl Acad Sci. 2011;108:20254-9.

47. Kilpatrick LA, Suyenobu BY, Smith SR, et al. Impact of mindfulness-based stress reduction training on intrinsic brain connectivity. Neuroimage. 2011:56:290-8.

48. Shehzad Z, Kelly AMC, Reiss PT, et al. The resting brain: unconstrained yet reliable. Cereb Cortex. 2009;19:2209-29.

49. Zuo XN, Kelly C, Adelstein JS, et al. Reliable intrinsic connectivity networks: test-retest evaluation using ICA and dual regression approach. Neuroimage. 2010;49:2163-77.

50. Radloff L. The CES-D scale: a self-report depression scale for research in the general population. Appl Psychol Meas. 1977:1:385-401.

51. Cohen S, Kamarck T, Mermelstein R. A global measure of perceived stress. J Health Soc Behav. 1983;24:385-96.

52. Spielberger CD, Gorsuch RL, Lushene PR, et al. Manual for the State-Trait Anxiety Inventory. Palo Alto, CA: Consulting Psychologists Press, 1983

53. Spielberger C. State-Trait Anger Expression Inventory-2, Professional Manual. Psychol Assess Resour Inc., 1999:1-7.

54. Diener E, Emmons RA, Larsen RJ, et al. The satisfaction with life scale. J Pers Assess. 1985;49:71-5. 
55. Gross JJ, John OP. Individual differences in two emotion regulation processes: implications for affect, relationships, and well-being. J Pers Soc Psychol. 2003;85:348-62.

56. Stunkard AJ, Messick S. The three-factor eating questionnaire to measure dietary restraint, disinhibition and hunger. J Psychosom Res. 1985;29:71-83.

57. Niemeier HM, Phelan S, Fava JL, et al. Internal disinhibition predicts weight regain following weight loss and weight loss maintenance. Obes (Silver Spring). 2007;15:2485-94.

58. Paffenbarger RS Jr, Blair SN, Lee IM, et al. Measurement of physical activity to assess health effects in free-living populations. Med Sci Sport Exerc. 1993:25:60-70.

59. Buysse DJ, Reynolds CF, Monk TH, et al. The Pittsburgh sleep quality index: a new instrument for psychiatric practice and research Psychiatry Res. 1989;28:193-213.

60. Anthropometry procedures manual. Centers for Disease Control and Prevention. National Health and Nutrition Examination Survey (NHANES), 2007.

61. Devilly GJ, Borkovec TD. Psychometric properties of the credibility/ expectancy questionnaire. J Behav Ther Exp Psychiatry 2000;31:73-86.

62. Baer RA, Smith GT, Lykins E, et al. Construct validity of the five facet mindfulness questionnaire in meditating and nonmeditating samples. Assessment. 2008;15:329-42.

63. Cohen J. A power primer. Psychol Bull. 1992;112:155-9.
64. Frison L, Pocock SJ. Repeated measures in clinical trials: analysis using mean summary statistics and its implications for design. Stat Med. 1992;11:1685-704

65. Rabe-Hesketh S, Skrondal A. Multilevel and longitudinal modeling using Stata. Am Statistician 2006;60:293-4.

66. Baron RM, Kenny DA. The moderator-mediator variable distinction in social psychological research: conceptual, strategic, and statistical considerations. J Pers Soc Psychol. 1986;51:1173-82.

67. MacKinnon DP. Introduction to statistical mediation analysis. Mahwah, NJ: Erlbaum; 2008.

68. Talairach J, Tournoux P. Co-Planar Stereotaxic Atlas of the Human Brain: 3-Dimensional Proportional System: an Approach to Cerebral Imaging. Hardcover 1988;39.

69. Genovese CR, Lazar NA, Nichols T. Thresholding of statistical maps in functional neuroimaging using the false discovery rate. Neuroimage. 2002;15:870-8.

70. Tzourio-Mazoyer N, Landeau B, Papathanassiou D, et al. Automated anatomical labeling of activations in SPM using a macroscopic anatomical parcellation of the MNI MRI single-subject brain. Neuroimage. 2002;15:273-89.

71. Wing RR, Tate DF, Gorin AA, et al. A self-regulation program for maintenance of weight loss. $N$ Engl J Med. 2006;355:1563-71. 